\title{
Lipid membranes on a surface grating studied by neutron reflectometry
}

\author{
C. Ollinger, D. Constantin, J. Seeger and T. Salditt \\ Institut fuer Roentgenphysik - Geiststrasse 1137073 Goettingen, Germany
}

PACS. 87.16.Dg - Membranes, bilayers, and vesicles.

PACS. 61.12.Ha - Neutron reflectometry.

PACS. 62.20.Dc - Elasticity, elastic constants.

\begin{abstract}
This experiment presents a new approach to measure elasticity parameters of lipid bilayers. We have deposited a stack of phospholipid bilayers on a silicon surface grating defined by e-beam lithography. The periodic surface profile acts as a boundary condition which imposes a controlled strain in the smectic film. Probing this distortion field by neutron reflectometry, we determine the smectic penetration length $\Lambda=\sqrt{K / B}$ from the relative intensity of the satellite peaks.
\end{abstract}

Introduction. - Elastic parameters such as bending stiffness and compressibility are important physical parameters for the understanding of amphiphilic assemblies. The elasticity properties of biomimetic lipid membranes in the fluid $L_{\alpha}$ phase are of particular interest [1-3] and can be probed in equilibrium stacks of bilayers or bulk phases of multilamellar vesicles with lamellar periodicity $d$. Such multilamellar systems of smectic-A liquid crystalline symmetry can be described by the linearized smectic free energy functional (Hamiltonian) $[4,5]$,

$$
H=\int_{A} d^{2} r \sum_{n=1}^{N-1}\left(\frac{1}{2} \frac{B}{d}\left(u_{n+1}-u_{n}\right)^{2}+\frac{1}{2} \kappa\left(\nabla_{x y}^{2} u_{n}\right)^{2}\right),
$$

where $\kappa$ denotes the bilayer bending rigidity, $A$ the area in the $x y$-plane, $N$ the number of bilayers, and $u_{n}$ the deviation from the average position $n d$ of the $n$th bilayer. $B$ and $K=\kappa / d$ are elastic coefficients, governing the compressional and bending modes of the smectic phase, respectively. A fundamental length scale in these systems is given by the smectic penetration length $\Lambda=\sqrt{K / B}$. A method to measure $\Lambda$ in smectics is given by the threshold of the layer buckling instability under dilation [6]. For stacks of lipid membranes, measurements of $\Lambda$ reported in the literature have used $\mathrm{x}$-ray scattering and lineshape analysis carried out on aqueous (bulk) suspensions [3], only if $\kappa \simeq k T$ is very small, and undulation modes are very strong. Contrarily, aligned lipid bilayers allow a separate determination of both parameters $K$ and $B[7,8]$. In both cases, thermal fluctuations of the bilayer height profiles are probed and analyzed with regard to Eq.(1). If now, the bilayers are rather stiff with $\kappa \gg k T$ as is the case for standard phospholipids such as DMPC [9] it can become difficult to probe the (c) EDP Sciences 
thermal diffuse scattering since the fluctuation amplitudes decrease and the scattering may easily be dominated by the signal of static defects.

In this Letter, we present an alternative approach to measure $\Lambda$ by an imposed static corrugation, without relying on thermal fluctuations. As can be shown by minimizing Eq.(1) subject to an adequate boundary condition [12], corrugations in a smectic system propagate along the stacking direction with a damped amplitude. The damping varies quadratically with the lateral wavevector $k_{i}$ corresponding to each Fourier mode, as $\exp \left(-z \Lambda k_{i}^{2}\right)$. The damping of the profile along $z$ within the stack is then measured by nonspecular neutron scattering which is sensitive to the height correlation functions of the buried lipid water interfaces. Instead of a sinusoidal profile with only one Fourier component we use a rectangular grating profile to simultaneously measure the damping of several Fourier coefficients separated in reciprocal space by the momentum transfer $q_{x}$ parallel to the plane of the bilayers.

Materials and methods. - (100)-silicon substrates (Silchem, Germany) were patterned using e-beam lithography and reactive ion etching. After cleaning the 2" silicon wafer (thickness $635 \mu \mathrm{m}$ ) in methanol and ultra-pure water (Millipore, Bedford, MA), poly-methylmethacrylate (PMMA) was spin-coated yielding a thickness of about $50 \mathrm{~nm}$ on the silicon as a resist. The wafer was exposed to an unfocused electron beam (LION LV1, Leica, Jena, Germany), defining a one-dimensional grating pattern, over a total area of $1.8 \times 2 \mathrm{~cm}^{2}$ in the PMMA layer. After developing in a mixture of (4:1) ethylenglycolmonobutylether and ethylenglycolmonoethylether, the grating was etched for 12 seconds in $\mathrm{CBrF}_{3}$ atmosphere at a pressure of $2 \mathrm{~Pa}$ and a flow of $10 \mathrm{sccm}$, using a home-built reactive ion etch (RIE), leading to a grating depth of about $\mathrm{h}=2 \mathrm{~nm} \pm 0.2 \mathrm{~nm}$, as determined by AFM. The unexposed PMMA was then removed by acetone, and the silicon grating was cleaned by subsequent washing in methanol and ultra-pure water, resulting in a hydrophobic surface, before deposition of the lipid membranes. A scanning electron microscopy (SEM) micrograph of the grating after the etching process (but before removal of the unexposed PMMA) is shown in Fig. $1 \mathbf{B}$, using the lithography system in SEM mode. 1,2-dimyristoyl-sn-glycero-3-phosphatidylcholine (DMPC) was bought from Avanti, Alabama, and was used without further purification. Multilamellar bilayers were prepared by spreading from organic solvent on the silicon grating. The lipid was dissolved in a (1:1) trifluoroethanol-chloroform mixture at a concentration of $20 \mathrm{mg} / \mathrm{ml}$. A drop of $1 \mathrm{ml}$ was then carefully spread onto the well-levelled and cleaned substrate, yielding a film thickness of about $D \simeq 16 \mu \mathrm{m}$, which corresponds to about $N \simeq 2900$ layers in the stack. After a slow evaporation process avoiding film rupture, remaining traces of solvent in the sample were removed by exposing the sample to vacuum over night. No difference in the wetting behavior compared to the preparation on flat surfaces was observed.

During the experiment the sample was mounted in a temperature-controlled chamber. A $1 \mathrm{~cm}$ thick silicon block (Siliziumbearbeitung A. Holm, Germany) is used as a transparent window for the neutron beam, which enters and leaves the block from the side (see Fig. $1 \mathrm{C}$ ). The sample was fixed on top of the block facing the solution. To reach the biologically relevant $L_{\alpha}$-phase, the sample was kept at a temperature of $45^{\circ} \mathrm{C}$ during the experiment, in a solution of 14.2 wt\% polyethyleneglycol (PEG) in $\mathrm{D}_{2} \mathrm{O}$ with a molar mass of 20000 (PEG 20000) leading to an osmotic pressure of about $1.8 \cdot 10^{5} \mathrm{~Pa}$ [13]. This osmotic pressure serves two purposes: Firstly, it suppresses strong thermal fluctuations, so that the static distortion field is the leading contribution to the diffuse scattering (at the relevant positions in reciprocal space, see below). Secondly, the osmotic pressure prevents or reduces thermal unbinding [14] of the lipid layers during the experiment. Note that the orientational alignment of the multilamellar stack with respect to the substrate (mosaicity) was better than $0.038^{\circ}$, which was the resolutiondominated FWHM-width of the specular peak. The pressure-distance relationship for DMPC 


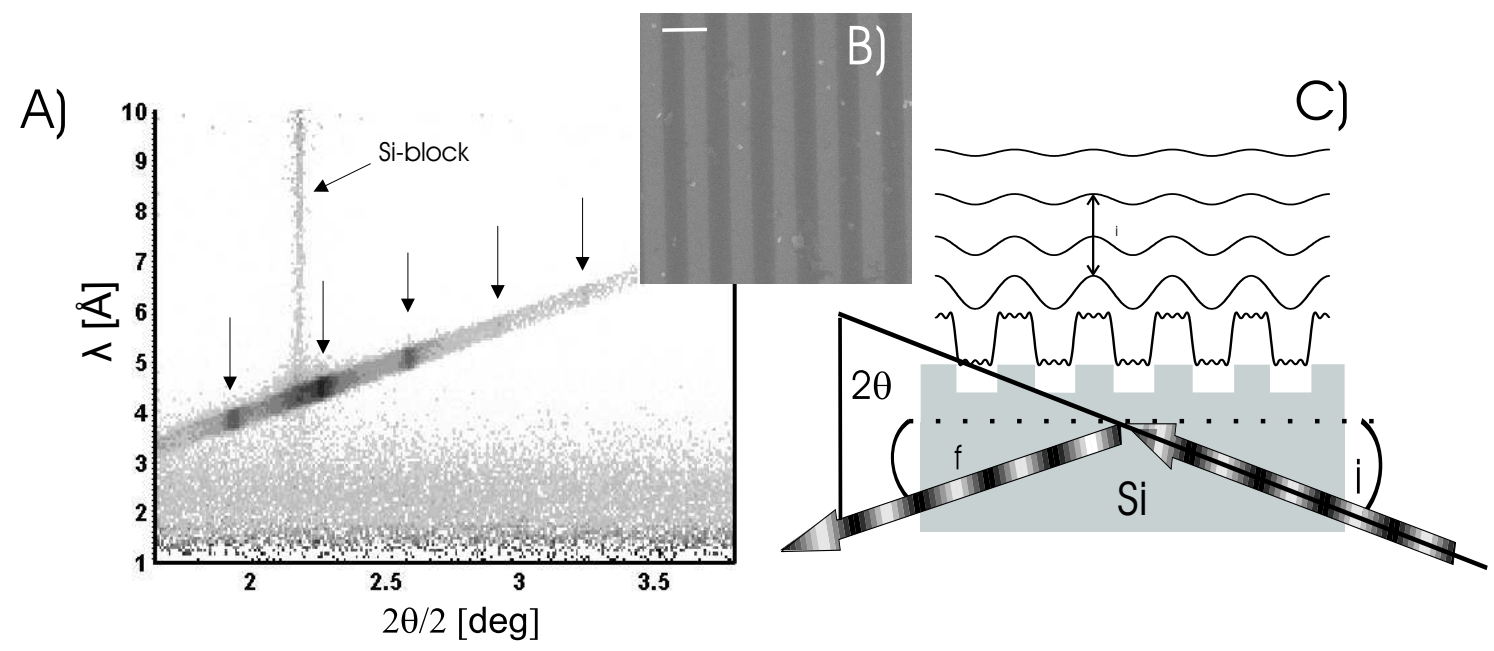

Fig. 1 - A) Measured intensity matrix $\mathrm{I}[2 \theta / 2, \lambda]$ of the lipid membrane stack deposited on the grating in logarithmically scaled gray shades. B) SEM micrograph of the silicon surface grating (top view) with $1 \mu \mathrm{m}$ scale bar. C) Sketch of the scattering geometry with the beam path through the silicon block and with the distortion field of the lipid bilayers as calculated by smectic elasticity theory. $\xi_{i}=\frac{1}{\Lambda k_{i}^{2}}$ denotes the vertical correlation length of a Fourier mode $k_{i}$ in the membrane stack.

as calculated from the interaction potentials at this osmotic pressure results in an equilibrium distance which agrees very well with the measured value $d=56.3 \AA$ [15]. Furthermore, thermal fluctuations are known to be significantly reduced at these pressures.

Experimental. - The experiments were carried out at the D17 reflectometer at the Institut Laue Langevin (ILL), Grenoble, in time-of-flight mode (TOF) [16]. Thermal neutrons were reflected off the sample and recorded as a function of exit angle (detector pixel) and wavelength $\lambda$ at different angles of incidence $\alpha_{i}$. Using the multiwire detector, the broad wavelength distribution, and the TOF electronic signal treatment, the specular and nonspecular (diffuse) reflectivity can thus be measured at a single value of $\alpha_{i}$, without scanning of motors. The sample was mounted vertically with the scattering vector in the horizontal $x z$-plane. The collimating slits were set to $0.55 \mathrm{~mm}$ (horizontal) at the sample position. In optimize intensity at the expense of $\lambda$ resolution a chopper opening of $5^{\circ}$ and rotational speed of $1000 \mathrm{rpm}$ was used. A multiwire proportional counter with $285 \times 285$ pixels (delay-line, Gabriel, EMBL Grenoble) was positioned $3.4 \mathrm{~m}$ behind the sample, equipped with an evacuated flight path. In TOF mode, the intensity matrix is obtained as follows: First, the counts are registered in each spatial pixel $(p x, p y)$ for 500 TOF channels. Next, the counts are integrated for fixed $p x$ and TOF channel along the vertical detector axis $p y$, yielding a $2 \mathrm{D}$ array of $\mathrm{I}[\mathrm{px}, \mathrm{TOF}$ channel]. The data is then read by an IDL routine, performing (i) the TOF $/ \lambda$ conversion, (ii) a correction for detector sensitivity (spatial variation of pixel sensitivity as a function of $p x$, as measured by incoherent scattering from $\mathrm{H}_{2} \mathrm{O}$ ), (iii) normalization by the $\lambda$-distribution, as measured by shots of the direct beam under identical chopper and slit settings, and (iv) a conversion from $p x$-pixel to scattering angle $2 \theta[17]$.

Fig. $1 \mathrm{~A}$ ) shows a typical $2 \mathrm{D}$ data set $\mathrm{I}[2 \theta / 2, \lambda]$ for DMPC in the fluid $L_{\alpha}$ phase at $T=45^{\circ}$ $\mathrm{C}$ deposited on the $1 \mu \mathrm{m}$ grating, at fixed $\alpha_{i}=2.2^{\circ}$, in logarithmically scaled grey shades. The different columns of the multiwire detector matrix correspond to different scattering angles $2 \theta$, 
with $2 \theta=\alpha_{i}+\alpha_{f}$. Rows of the intensity array are equivalent to the simultaneous measurement of a detector scan for each given $\lambda$. Enhanced diffuse scattering stemming from correlated corrugations induced by the grating in the multilamellar stack is observed at discrete positions (arrows) corresponding to the grating orders intersecting the first diffuse Bragg sheet (oblique line Fig $1 \mathbf{A}$ ) at constant $q_{z}=2 \pi / d$. A streak corresponding to the specular reflection of the supporting Si-block (chamber window) is observed at constant $2 \theta / 2=2.2^{\circ}$, slightly tilted with respect to the sample wafer fixed on top. Higher order Bragg sheets are not observed in the angular and wavelength range covered. Note that the satellite lattice peaks of the diffuse Bragg sheet reflect the contributions of the static deformation field. For a treatment of the diffuse scattering from pure thermal contribution (the Bragg sheet) by TOF neutron scattering we refer to [17]. Here we concentrate on the satellite peaks induced by the grating. In principle, the $q_{z}$-width of these satellites contains the information on the vertical correlation length of the static roughness, and thus also the smectic length scale $\Lambda$. However, since the intrinsic width cannot be resolved, we analyze the intensities of the lattice peaks, which reflect the relative strength of the Fourier coefficients averaged over the corrugated multilamellar stack. Since higher Fourier components decay faster than smaller ones with increasing separation form the surface, the coefficient $\Lambda$ can be evaluated from the intensity variations of the satellite peaks along the Bragg sheet. To analyze the data, the intensity was first integrated over the first Bragg sheet along $\lambda$ in the $\mathrm{I}[\theta, \lambda]$ dataset (Fig. $1 \mathbf{A}$ ). This is motivated by the fact that the width of the Bragg sheet $\left(\Delta q_{z} \approx 0.015 \AA^{-1}\right)$ was resolution limited in the present experiment. This integrated intensity curve $I_{\text {int }}(\lambda, 2 \theta / 2)$ is shown in Figure $2 \mathbf{A}$ as a function of the scattering angle $2 \theta / 2$. The data corresponds to a scattering distribution at constant $q_{z}=0.1115 \AA^{-1}$ over a varied $q_{x} \in\left[-1.2610^{-3} \AA^{-1}, 2.9810^{-3} \AA^{-1}\right]$. The experimental values are shown along with a six peak Lorentzian fit with linear background. Contrary to the peak widths, the integrated intensities (areas under the side peaks) are not affected by instrumental resolution. The integrated intensities were then normalized to the specular peak at $q_{x}=0$ and are displayed as a function of peak order in Fig. 2 B along with calculated peak intensities.

Data analysis and Simulation. - We consider the simplest model which can account for the intensity variation of the integrated Bragg sheet satellites (grating orders) with order number $n$, based on smectic elasticity theory. Since at the positions of the lattice orders, thermal diffuse scattering is a background contribution with respect to the dominating scattering from the grating corrugation, we can primarily consider the profile imposed by the boundary and damped out by the stack. An analytical treatment of nonspecular scattering based on [18] is quite complex for a multilamellar sample on a grating. Note that already a two interface problem (homogeneous thin film on grating) is difficult to solve [19], due to the non-linearity of the scattering theory. As a result of the non-linearity the intensity of the $n$th order is not proportional to the $n$th Fourier coefficient of the membrane height profile. We therefore choose an approach where we first numerically simulate the distorted layers on the grating, and then calculate the scattering by discrete points distributed on the corrugated stack. Each membrane is represented in the model by a line of point scatterers distributed on the calculated height profile. To model the grating-induced corrugations in the stack, a Fourier series including up to 7 coefficients $f_{i}$ was used to approximate the deformation of the layers. This was sufficient since experimentally only the orders $n \leq 3$ were observed. Following the smectic model, the damping of the Fourier coefficients varies with the lateral wavevector $k_{i}$ of each corrugation mode and the position $z$ of each layer in the stack, as $\exp \left(-z \Lambda k_{i}^{2}\right)$. The initial Fourier coefficients $f_{i}$ of the surface grating were obtained by a Discrete Fourier Transformation (DFT) of a step function with parameters $h, \nu$ and $a$ in real space. For the deviation $u(x, z)$ from the mean position $z=n d$ of each layer $n$, we can write 


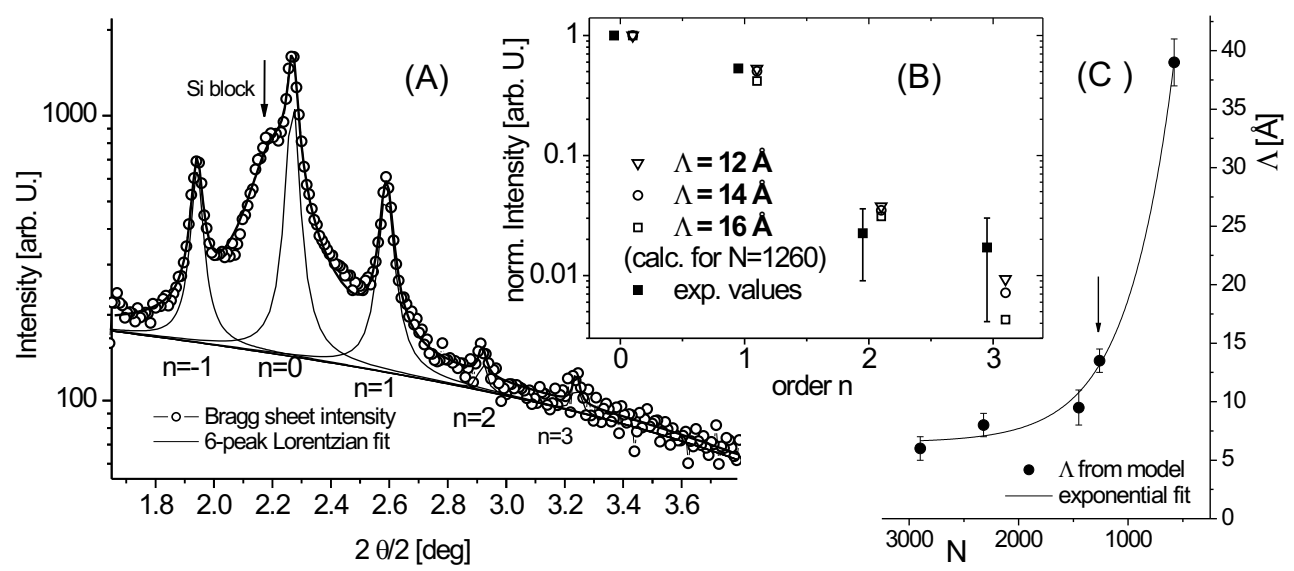

Fig. 2 - A $q_{z}$-integrated intensities of the first Bragg sheet (open circles) together with a 6 peak Lorentzian fit (solid line) B) Calculated peak areas for different values of $\Lambda$ (open symbols) and measured peak intensities (filled squares) normalized to specular peak $(n=0)$. C) Results for the parameter $\Lambda$ calculated numerically according to the model as a function of $N$. The arrow denotes the value of the experiment, after partial detachment of membranes.

$$
u(x, z)=\sum_{i} f_{i} \cos \left(k_{i} x\right) \exp \left(-z \lambda k_{i}^{2}\right)
$$

For the linear grating, the lipid membrane stack can be modelled in only two dimensions, $\mathrm{x}$ and $\mathrm{z}$, since the translation invariance in the $\mathrm{y}$-direction leads to a factor $\delta\left(q_{y}\right)$ in the scattering intensity. Scattering is described in the kinematical approximation. Each discretisation point in the stack is modelled as a point-scatterer and therefore the scattering intensity is described by

$$
I\left(q_{x}, q_{z}\right)=\left|\sum_{N} \sum_{x, z} \exp \left(-i(N d+u(x, z)) q_{z}\right) \exp \left(-i x q_{x}\right)\right|^{2} .
$$

The calculation was performed around the first Bragg sheet with range corresponding to a $q_{z}$-width of $0.015 \AA^{-1}$. The results were integrated along $q_{z}$ to obtain $I\left(q_{x}, q_{z}=2 \pi / d\right)$ and then compared to the measured intensities. Figure $2 \mathbf{B}$ ) shows the measured peak intensities compared to the calculation for different values of the smectic penetration length $\Lambda$. The preparation parameters (mass of lipid deposited per area) correspond to a number of layers $N \cong 2900$ in the stack. However, a ratio of 2.3 between the total intensities of the diffuse scattering (integrated over the entire angular range) before and after filling the sample cell with water, indicated a significant detachment of bilayers, in agreement with only about $N \simeq 1260$ bilayers. The lamellar periodicity $d=56.3 \AA$ was determined from the position of the first Bragg peak. The $x$-periodicity $a$ obtained from positions of the satellite peaks (Fig. 2 A) confirms the fabrication value of $a=1 \mu m . I\left(q_{x}\right)$ is sensitive to the amplitudes of all Fourier modes $f_{i}$, which are computed by Fourier synthesis of a step function with a 
step height $h=2 \mathrm{~nm}$, a line to space ratio of $\nu=1$ and a periodicity of $a=1 \mu \mathrm{m}$. The value for the step height $h$ is obtained from the etching process and confirmed by the AFM measurements. The value for $\nu$ is obtained from SEM measurements as shown in Figure 1 B. Two more numerical parameters were used in the simulation i.e. the number of grating periods in $x$-direction and the points per $x$-period. They were empirically set to 10 and 100 respectively in order to achieve a suitable resolution (width of the satellite peaks and number of points on each peak) in the calculated scattering pattern. Variation of these two parameters showed that discretisation errors were negligible and the precise choice of their values was not important, in particular since all simulated satellite peaks were afterwards integrated. Finally, the smectic penetration length $\Lambda$ defines the damping of each mode in the modelled membrane stack. The higher $\Lambda$, the stronger the damping, and the lower the satellite peak intensity.

Results and Conclusions. - The calculations were first performed for fixed value $N=$ 2900 as deposited, leading to a surprisingly small value of $\Lambda=(5.35 \pm 0.25) \AA$. In a second step, it was realized that a significant amount of bilayers had detached, most likely when filling the cell with the polymer solution. The loss of bilayers was evidenced by analyzing the total diffuse scattering (TDS) (integrated over the entire angular range) measured before and after filling the cell. The TDS is proportional to the number $N$. It was found that the TDS decreased by a factor of 2.3. Reiterating the calculation with $N=1260$ gives a result of $\Lambda=(12.4 \pm 0.7) \AA$, which is in much better agreement with the literature. The error on $\Lambda$ was determined by firstly calculating the range of theoretical values consistent with the experimental error interval of each order $n=1,2,3$, and secondly taking the corresponding and error-weighted average. A short remark concerning the interdependence of $\Lambda$ and $N$ in the model: $\Lambda$ can only be determined, if $N$ is known, since the intensity variation of the satellite depends on both parameters, as illustrated by Fig.2 C. Note that if $N$ is assumed small, the high Fourier components of the distortion field induced by the surface grating must decay faster (corresponding to a large $\Lambda$ ) in order to explain the experimental results. Importantly, by comparison of the total diffuse scattering before and after exposing the sample to polymer solution, the value of $N$ can be inferred. After correction for the loss of bilayers, we thus obtain a smectic length $\Lambda=(12.4 \pm 0.7) \AA$. Under the given conditions, a compressional modulus $B=4.5 \mathrm{MJ} / \mathrm{m}^{3}$ (DMPC, $d=56 \AA$ ) can be expected [15,20]. Together with this value, $\Lambda=12.4 \AA$ yields a bending stiffness of $\kappa=9.7 k_{B} T$, which is at the lower end of published values. Future improvements should aim at an improvement of the experimental resolution, and a variation of the film thickness in particular to smaller values of $N$. For thin stacks it should then become possible to measure directly the $q_{z}$ width of the satellites, from which the number of bilayers $N$ can be obtained. Finally, non-linear elastic effects could possibly start to play a role, in particular if the height of the grating is increased. The grating parameters should therefore be varied to investigate this issue further.

In summary, we have used the boundary condition of a lattice grating imposed on a stack of lipid membranes to probe the elastic response of the bilayer stack from the corresponding nonspecular diffraction, and to measure the smectic penetration length $\Lambda$, from which the bending rigidity can be inferred. More generally, macromolecular assemblies on controlled lithographic nanostructures may lead to novel experimental schemes for probing elasticity parameters and self-assembly properties. An extension to dynamic relaxation parameters could be achieved by a temporally varied surface corrugation field, e.g. induced by surface acoustic waves. 
We would like to thank G. Fragneto-Cusani and R. Cubitt for help at the D17 reflectometer, and J. Garleff for the AFM measurements. D. C. has been supported by a Marie Curie Fellowship of the European Community programme Improving the Human Research Potential under contract number HPMF-CT-2002-01903.

\section{REFERENCES}

[1] R. Lipowsky in Structure and Dynamics of Membranes, Vol 1, Parts A \& B, R. Lipowsky, E. Sackmann, eds. (Handbook of Biological Physics, 1995).

[2] H.I. Petrache et al., Phys.Rev.E 57, 7014 (1998).

[3] C.R. Safinya et al., Phys. Rev. Lett. 62 (1989) 1134; ibid 572718 (1986).

[4] A. Caillé, C.R.Acad.Sci. B 274891 (1972).

[5] N. Lei, C.R. Safinya, R. Bruinsma, J. Phys. II France, 5, 1155 (1995); N. Lei, Ph.D. Rutgers 1993.

[6] P. Oswald and P. Pieranski, Les Cristaux Liquides: Concepts et Propriétés Physiques Illustrés Par Des Expériences Vol. II, Gordon and Breach, Paris 2000.

[7] Lyatskaya Y. and LiU Y. and Tristram-Nagle S. and Katsaras J. and Nagle J.F., Phys.Rev. E, 63 (2000) 011907.

[8] Salditt, T. and Vogel, M. and Fenzl, W., Phys Rev. Lett., 90 (2003) 178101.

[9] Phospholipid bilayers typically exhibit a bending stiffness $\kappa$ of several $k_{B} T$, significantly larger than the values of single tail amphiphiles. For DMPC in the fluid phase published values range from $\kappa=32 k_{B} T[10]$ to $\kappa \simeq 11 k_{B} T$ [11].

[10] C.-H. LeE and W.-C. Lin and J. Wang, Physical Review E, 64 (2001) 020901.

[11] G. Pabst and J. Katsaras and A. Velayudhan and A. Raghunathan and M. Rappolt, Langmuir, 19 (2003) 1716-1722.

[12] P. G. DE Gennes, The Physics of Liquid Crystals (Clarendon, Oxford) 1974.

[13] The data was obtained from the web site of the Membrane Biophysics Laboratory at the Brock University in Canada : http://aqueous.labs.brocku.ca/osfile.html. The value for the osmotic pressure induced by a PEG 20000 solution at $14.2 \mathrm{wt} . \%$ is only available at $20 C$ as $1.910^{5} \mathrm{~Pa}$. At $45 C$, temperature at which the experiments were performed, we estimate that the pressure is lower by about 10 to $20 \%$, by using the temperature dependence of PEG 8000 solutions (available on the same site).

[14] M. Vogel et Al., Physical Review Letters, 84 (2000) 390.

[15] U. Mennicke, PhD Thesis, University Goettingen, Germany, (2003) ; U. Mennicke, D. Constantin, T. Salditt, sent to Phys.Rev.E .

[16] R. Cubitt and G. Fragneto, Appl. Phys. A, 74 (2003) 329.

[17] T. Salditt and C.Münster and U. Mennicke and C.Ollinger and G. Fragneto, Langmuir, 19 (2003) 7703.

[18] S.K. Sinha et al. Phys. Rev. B 38 (1988) 2297; S.K. Sinha, J.Phys.III (France) 4 (1994).

[19] M. Tolan ET AL., Jounal of Physics D: Applied Physics, 28 (1995) A231

[20] H. I. Petrache and N. Gouliaev and S. Tristam-Nagle and R. Zhang and R. M. Suter and J. F. NAGLe, Physical Review E, 57 (1998) 7014 